\title{
Oscillatory Beta Activity Mediates Neuroplastic Effects of Motor Cortex Stimulation in Humans
}

\author{
Craig J. McAllister, Kim C. Rönnqvist, Ian M. Stanford, Gavin L. Woodhall, Paul L. Furlong, and Stephen D. Hall \\ Aston Brain Centre, School of Life and Health Sciences, Aston University, Birmingham, B4 7ET, United Kingdom
}

Continuous theta burst stimulation (cTBS) is a repetitive transcranial magnetic stimulation protocol that can inhibit human motor cortex (M1) excitability and impair movement for $\leq 1 \mathrm{~h}$. While offering valuable insights into brain function and potential therapeutic benefits, these neuroplastic effects are highly variable between individuals. The source of this variability, and the electrophysiological mechanisms underlying the inhibitory after-effects, are largely unknown. In this regard, oscillatory activity at beta frequency $(15-35 \mathrm{~Hz})$ is of particular interest as it is elevated in motor disorders such as Parkinson's disease and modulated during the generation of movements. Here, we used a source-level magnetoencephalography approach to investigate the hypothesis that the presence of neuroplastic effects following cTBS is associated with concurrent changes in oscillatory M1 beta activity. M1 cortices were localized with a synthetic aperture magnetometry beamforming analysis of visually cued index finger movements. Virtual electrode analysis was used to reconstruct the spontaneous and movement-related oscillatory activity in bilateral M1 cortices, before and from 10 to $45 \mathrm{~min}$ after cTBS. We demonstrate that $40 \mathrm{~s}$ of cTBS applied over left M1 reduced corticospinal excitability in the right index finger of 8/16 participants. In these responder participants only, cTBS increased the power of the spontaneous beta oscillations in stimulated M1 and delayed reaction times in the contralateral index finger. No further changes were observed in the latency or power of movement-related beta oscillations. These data provide insights into the electrophysiological mechanisms underlying cTBS-mediated impairment of motor function and demonstrate the association between spontaneous oscillatory beta activity in M1 and the inhibition of motor function.

\section{Introduction}

Non-invasive brain stimulation techniques such as repetitive transcranial magnetic stimulation (rTMS) can induce sustained neuroplastic effects and change motor behavior (for review, see Hoogendam et al., 2010). These techniques are therefore of great interest in relation to understanding and optimizing neurorehabilitation following brain injury or stroke or in movement disorders such as Parkinson's disease (PD) (Ridding and Rothwell, 2007). Patterned stimulation protocols are considered to be particularly promising as the application of $40 \mathrm{~s}$ of continuous theta burst stimulation (cTBS) over the human primary motor cortex (M1) has been reported to produce a strong inhibition of cortical excitability and impairment of motor function for up to $1 \mathrm{~h}$ (Di Lazzaro et al., 2005; Huang et al., 2005). However, not all participants display such profound inhibitory aftereffects (McAllister et al., 2011; Goldsworthy et al., 2012; Hamada et al., 2012) and

\footnotetext{
Received Dec. 9, 2012; revised March 11, 2013; accepted March 12, 2013.

Author contributions: C.J.M. and S.D.H. designed research; C.J.M. and K.C.R. performed research; C.J.M. and S.D.H. analyzed data; C.J.M., K.C.R., I.M.S., G.L.W., P.L.F., and S.D.H. wrote the paper.

This study was supported by funding from the Biotechnology and Biological Sciences Research Council, the Aston Research Centre for Healthy Ageing, and an RCUK fellowship (S.D.H.). The Wellcome Trust Laboratory for MEG Studies at the Aston Brain Centre is supported by the Wellcome Trust and the Dr. Hadwen Trust for Humane Research. Structural MRI scans were supported by the Lord Dowding Fund for Humane Research. We are very grateful to Dr. Caroline Witton and Dr. Martin McDonagh, as well as to two anonymous referees, for carefully reading this manuscript and their many suggestions that helped improve this paper.

This article is freely available online through the J Neurosci Author Open Choice option.

Correspondence should be addressed to Dr. Craig McAllister, Aston Brain Centre, School of Life and Health Sciences, Aston University, Birmingham, B4 7ET, UK. E-mail: c.mcallister@aston.ac.uk.

DOI:10.1523/JNEUROSCI.5624-12.2013

Copyright $\odot 2013$ the authors $\quad 0270-6474 / 13 / 337919-09 \$ 15.00 / 0$
}

recent outcomes in stroke and $\mathrm{PD}$ patients have proved disappointing (Eggers et al., 2010; Talelli et al., 2012). An improved understanding of the underlying mechanisms of TBS-mediated effects and the factors affecting its variability is, therefore, essential to advance its therapeutic value.

The inhibitory after-effects of cTBS are typically proposed to involve long-term depression-like changes in synaptic transmission as described in animal models (Hess and Donoghue, 1996). The extent to which they are related to changes in cortical oscillatory activity is rarely examined; however, and the few studies to do so, using electroencephalography (EEG) recordings, have produced contrasting results (McAllister et al., 2011; Noh et al., 2012). The lack of attention to this topic is rather surprising considering that activity in human M1 is characterized by spontaneous neuronal network oscillations at a beta-band $(15-35 \mathrm{~Hz})$ frequency. Furthermore, electrophysiological studies in PD patients show a strong relationship between impaired movement and elevated oscillatory beta activity within the motor system (Brown, 2007).

Stagg et al. (2009) demonstrated, using magnetic resonance spectroscopy (MRS), that cTBS increased GABA concentration within the stimulated M1 region. Studies in animal models and humans indicate that beta oscillations are the summed output of principal cells temporally aligned by GABAergic interneuron rhythmicity (Jensen et al., 2005; Yamawaki et al., 2008). The power of both spontaneous and movement-related oscillatory beta activity in human M1 are GABA-A dependent, as demonstrated by their modulation following the administration of benzodiazepine drugs (Jensen et al., 2005; Hall et al., 2010, 2011). 
Therefore, if increased GABAergic drive underlies the inhibitory after-effects of cTBS on the human motor system, it is expected that it will be reflected in concurrent changes in spontaneous and/or movement-related beta activity.

Here, we exploited the high spatial and temporal resolution of contemporary magnetoencephalography (MEG) methods to determine the impact of cTBS on spontaneous and movementrelated oscillatory activity in M1 cortices. We demonstrate an association between increased spontaneous oscillatory beta activity and the presence of cTBS-mediated inhibitory after-effects, thereby strengthening our understanding of the mechanisms underlying CTBS and the functional role of beta oscillations in the human motor system.

\section{Materials and Methods}

\section{Subjects}

Sixteen right-handed healthy volunteers (aged 19-44 years, nine females and seven males) with normal or corrected to normal vision participated in the study. The TMS Adult Safety Screen (Keel et al., 2001) was used to identify any participants who may have been predisposed to possible adverse effects of the stimulation. No adverse effects of TMS occurred during the course of the experimental procedures. The study was performed in accordance with the Declaration of Helsinki, and approved by the Ethics Committee of the School of Life and Health Sciences at Aston University. Written informed consent was obtained from all participants.

\section{Electromyography recording and TMS}

The electromyographic (EMG) activity of the first dorsal interosseous (FDI) muscle in the right hand was recorded using bipolar, single differential surface EMG electrodes (DE-2.1; Delsys). The surface electrodes comprised two $10 \times 1 \mathrm{~mm}$ silver bar strips, spaced $10 \mathrm{~mm}$ apart, with a 20 $\mathrm{Hz}-450 \mathrm{kHz}$ bandwidth, $92 \mathrm{~dB}$ common mode rejection ratio, and $>10^{15} \Omega$ input impedance. The electrodes were placed over the muscle and a reference ground electrode was placed over the ulnar process of the right wrist. The EMG signal was digitized with a sampling rate of $2 \mathrm{kHz}$ using a Micro 1401 analog-digital converter and analyzed using Signal version 4 (Cambridge Electronic Design).

Single-pulse TMS was performed using a Magstim $200^{2}$ stimulator and cTBS was performed using a Magstim Super Rapid stimulator (Magstim). Both forms of stimulation were performed using a $70 \mathrm{~mm}$ diameter figure-of-eight coil, held tangentially to the scalp with the coil handle pointing backward $\sim 45^{\circ}$ laterally. The optimal position for evoking a response in the FDI muscle was marked on the scalp and the coil position was then fixed using a mechanical arm (Manfrotto \& Co). Active motor threshold (AMT) was defined as the minimum stimulator output necessary to evoke a response of at least $200 \mu \mathrm{V}$ in 5 of 10 consecutive trials from the FDI muscle as participants used visual feedback to maintain a force level corresponding to $\sim 5 \%$ maximum voluntary contraction. Participants were instructed to open their eyes and to relax their hand and finger muscles during all other TMS procedures.

\section{$M E G$}

Neuromagnetic activity was recorded using a whole-head 275 channel MEG system (VSM Medtech) in a magnetically shielded room. Participants were seated with eyes open and observed a central fixation point on a computer monitor at a distance of $1.5 \mathrm{~m}$. Three fiducial coils were affixed to each participant at the nasion and bilateral pre-auricular points to continuously monitor head position during recordings. A 3D digitization of each participant's scalp and fiducial coil positions (Polhemus Isotrak; Kaiser Aerospace), were coregistered with their $3 \mathrm{~T}$ anatomical MR image (Siemens) using a modified version of a previously used surface-matching algorithm (Adjamian et al., 2004). Data were collected at a sample rate of $1200 \mathrm{~Hz}$ using a third-order gradiometer configuration with a $50 \mathrm{~Hz}$ notch filter and $1-300 \mathrm{~Hz}$ bandpass filter.

\section{Experimental protocols}

In the first experimental session, single-pulse TMS was used to assess changes in corticospinal excitability within each participant before and after the application of a $40 \mathrm{~s}$ train of cTBS over left M1. Corticospinal excitability was assessed by measuring the mean amplitude of the motorevoked potential (MEP) in the contralateral FDI muscle elicited by 15 test pulses, delivered every $4.0-6.0 \mathrm{~s}$. The stimulus intensity was individually adjusted for each participant so that the mean amplitude of the peakpeak MEP in the pre-TBS recording ranged between 0.5 and $1 \mathrm{mV}$ (Cheeran et al., 2008). cTBS was then applied for 40 s over left M1 using the protocol of Huang et al. (2005) in which three pulses at $50 \mathrm{~Hz}$ are applied every $200 \mathrm{~ms}$ (see Fig. $4 \mathrm{~A}$ ). The stimulus intensity was set at $80 \%$ AMT and ranged from 28 to $44 \%$ maximal stimulator output across participants. Corticospinal excitability was then measured at $5 \mathrm{~min}$ intervals from 5 to $45 \mathrm{~min}$ post-TBS as the mean peak-to-peak amplitude of 15 MEPs that were elicited with the same intensity used in the pre-TBS period. Any trial displaying high EMG activity $(>20 \mu \mathrm{V})$ in the $200 \mathrm{~ms}$ before the onset of the TMS pulse was discarded off-line.

Muscle contractions performed before and during cTBS have been shown to influence the subsequent effect on corticospinal excitability (Gentner et al., 2008; Huang et al., 2008). We were, therefore, careful to ensure that the motor behavior of each participant was standardized in the 10 min before cTBS. To obtain individual AMT measurements, participants performed 3-4 isometric contractions of the FDI muscle, each of $\sim 1$ min duration. cTBS was then performed $\sim 5-6$ min after the end of the last isometric contraction. This allowed time for the coils to be switched between the Magstim Super Rapid and Magstim $200^{2}$ stimulators and for the pre-TBS measurement of corticospinal excitability. Participants were requested to remain still and to keep their hands relaxed during all of these procedures and compliance was monitored using the EMG recordings.

The second experimental session was completed at least $72 \mathrm{~h}$ after the first session but at the same time of day. Here we used MEG recordings to assess the effect of an identical $40 \mathrm{~s}$ train of cTBS on spontaneous and movement-related oscillatory activity in bilateral M1. Participants were first positioned in the MEG system, where they performed a protocol, consisting of $3 \times 1$ min rest periods and two blocks of 30 finger movement trials lasting $\sim 180 \mathrm{~s}$ each. In each movement block, either left only or right only finger abduction was required as quickly as possible in response to a change in fixation cross color, which occurred with an interstimulus interval of $6 \mathrm{~s}$ and a randomized jitter of $\pm 500 \mathrm{~ms}$. Movement timings were measured using both a button press response and EMG recordings. A primary somatosensory (S1) index finger localizer stimulus, consisting of $2 \times 100$ s periods of mild electrical stimulation, was delivered to the left and right index fingers at the end of the recording block (data not shown). Following completion of the pre-TBS MEG recording, participants were removed from the scanner and AMT was first recorded using the same procedure as the first experimental session. Forty seconds of cTBS was then applied over left M1, after which participants were returned to the MEG system to complete two more experimental protocols identical to that described above. Participants were requested to keep their hands and arms as still as possible while being helped into the MEG system. The "post-TBS1" MEG recording began 10 min after stimulation and the "post-TBS2" MEG recording began $30 \mathrm{~min}$ after stimulation.

\section{Data analysis}

TMS corticospinal excitability. In each participant, the mean peak-topeak MEP amplitude was calculated in each block of 15 trials, which was used to determine the effects of cTBS on corticospinal excitability. Participants could be clearly separated into two groups based upon their pre- to post-TBS change in corticospinal excitability. Based upon the terminology used by Hamada et al. (2012), those subjects who displayed a mean MEP reduction $>10 \%$, as averaged across all post-TBS recordings, were categorized as "responders" and those that showed no change or a facilitation of MEP amplitude were categorized as "nonresponders." For comparison with MEG measures, these data were then grouped into three blocks: (1) pre-TBS, comprising the baseline period before cTBS; (2) post-TBS1, comprising the 10-25 min after cTBS; and (3) post-TBS2, comprising 30-45 min after cTBS.

Calculation of reaction time. Thirty reaction-time trials were recorded from both the left and right hand during each of the three MEG recording 
A

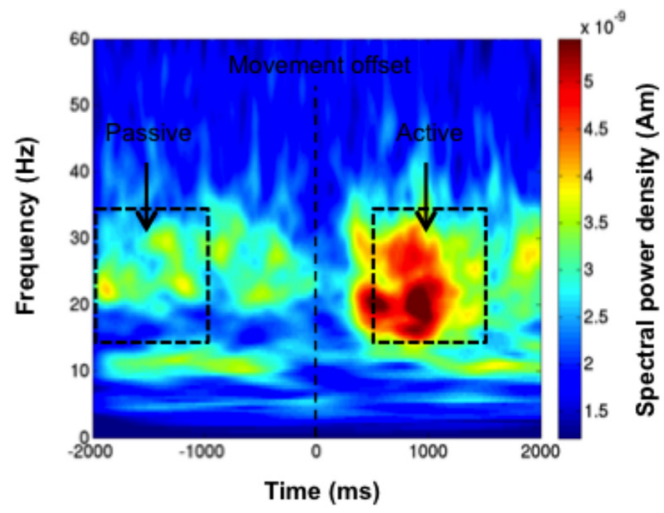

B
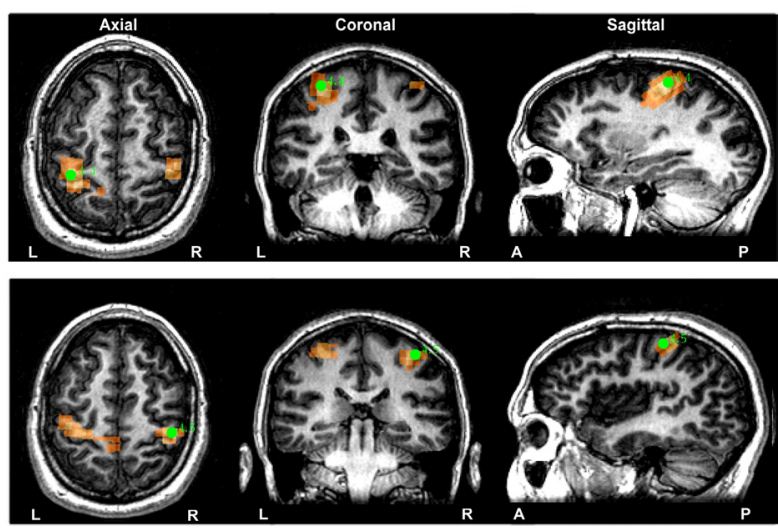

Figure 1. Localization of motor cortex. A, Time-frequency plot of the source activity from a virtual sensor in a representative participant as derived from the SAM beamformer approach. The location was the largest PMBR activity in left M1 during abductions of the right index finger. Trials were aligned to the point of movement offset. The passive $(-2.0$ to $-1.0 \mathrm{~s})$ and active $(0.5-1.5 \mathrm{~s})$ time periods used to create the differential SAM images are indicated by black boxes. $B$, Functional identification of left M1 and right M1 from the PMBR following abductions of the contralateral index fingers. The 3D coordinates (green circles), which are superimposed upon the participant's MR image, represent the maximal $t$ scores in left M1 (top, $t=4.4$ ) and right M1 (bottom, $t=4.5$ ), and indicate the neural generators of the PMBR.

blocks (pre-TBS, post-TBS1, and post-TBS2). Reaction times were calculated from the onset of the visual cue to the onset of the EMG activity in the FDI muscle. Trials containing reaction times $<100 \mathrm{~ms}$ or $>3$ SDs slower than the participants mean reaction time were discarded from the analysis ( $2 \%$ of total). Trials containing large artifacts in the MEG signal were also excluded from further analysis (3\% of total).

\section{MEG analysis}

Source localization of motor cortex. Bilateral M1 were localized using the established Synthetic Aperture Magnetometry (SAM) beamforming method (Veen et al., 1997; Vrba and Robinson, 2001). This approach computes a difference image, using the pseudo $t$ statistic of the change in predefined oscillatory power across all voxels within the anatomical MRI-determined source space (Hillebrand et al., 2005). Here, with timezero defined as the offset of EMG activity associated with each index finger abduction (termed movement offset) we localized the source of the post-movement beta rebound (PMBR; Fig. 1A). This is achieved by computing a difference image of the beta $(15-35 \mathrm{~Hz})$ frequency power between a pre-movement period $(-2.0$ to $-1.0 \mathrm{~s})$ and postmovement period $(0.5-1.5 \mathrm{~s})$. Using this approach, the $3 \mathrm{D}$ coordinates of the maximal $t$ score is a reflection of the neural generator of the PMBR, which is robustly localized to the M1 hand area contralateral to the side of the finger movement (Jurkiewicz et al., 2006; Hall et al., 2011). The maximal $t$ value in the sensorimotor cortex region provided the functional identification of bilateral M1 in each participant (Fig. 1B).

Estimation of spontaneous and functional oscillatory power. Data analyses were performed using custom software routines written using MATLAB (MathWorks). The effect of cTBS on spontaneous beta oscillatory power from bilateral M1 was assessed from three $60 \mathrm{~s}$ rest periods within each MEG recording block. Muscle activity during the rest periods may have had a confounding influence on the spontaneous beta oscillatory activity. Data were therefore screened using an automated process, in which MEG data obtained during resting EMG activity that exceeded 30 $\mu \mathrm{V}$ were excluded from further analysis. For each participant, a spatially constrained implementation of the SAM beamformer was used, in which optimal sensor weights are computed and summed for a specific source location, offering an optimal spatial filter for the region of interest; a so-called "virtual electrode" (Robinson and Rose, 1992). Here, the virtual electrodes were reconstructed specifically for left and right M1 based upon the coordinates identified by SAM analysis of PMBR. The power profile of spontaneous oscillatory activity was determined using Morlet wavelet time-frequency analysis of the virtual electrode output over the $1-50 \mathrm{~Hz}$ range in frequency bins of $0.5 \mathrm{~Hz}$ (Fig. 2A). Power spectral density plots were then created for each $60 \mathrm{~s}$ rest period (Fig. $2 B$ ). The peak amplitudes of the spontaneous oscillatory power within both muand beta-band frequencies were obtained from each 60s rest period. These values were then averaged across each of the MEG recording blocks (pre-TBS, post-TBS1, and post-TBS2) for both left and right M1.

Virtual electrode data from the same M1 loci were then used to determine the effects of cTBS on movement-related beta oscillations. The movement trials from each MEG recording were first aligned from -1.0 to $+1.0 \mathrm{~s}$ around the onset of the visual go-cue (Fig. $3 A$ ). The average movement-related spectral power was then reconstructed for this time period using a Morlet wavelet-based technique described above (Jurkiewicz et al., 2006). The baseline beta power was calculated as the mean amplitude during the $900 \mathrm{~ms}$ before the visual cue onset (Fig. 3C, BP1). The onset of the movement-related beta desynchronization (MRBD) was also determined as the first point following the visual cue in which the beta band power fell $<2.5$ SDs of the power in the baseline period (Fig. 3C).

The movement trials from each MEG recording were then realigned from -1.0 to $+1.0 \mathrm{~s}$ around the movement onset, which was determined as the onset of EMG activity in the right index finger (Fig. 3B). The average movement-related spectral power was then reconstructed for this time period using the same approach described above. The amplitude of the beta power at the onset of EMG activity (Fig. 3D, BP2) was recorded from each average. This provided a measurement of the MRBD that occurred before the onset of the movement. The magnitude of the MRBD was calculated as the mean beta power measured during the baseline period (BP1) minus that at the point of EMG onset (BP2). This was converted to a percentage change by dividing by the baseline power (BP1) and multiplying by 100 .

To further determine the effects of cTBS on movement-related oscillatory activity, the following three measures were taken from the right index finger movement trial of the responder participants only: (1) " $\Delta$ RT" calculated as the reaction time in the post-TBS1 recording minus the reaction time in the pre-TBS recording; positive values indicated that reaction times were slower in the post-TBS1 recording. (2) " $\triangle \mathrm{MRBD}$ magnitude" calculated as the percentage MRBD in the post-TBS1 recording minus the percentage MRBD in the pre-TBS recording; positive values indicated that the relative magnitude of the MRBD was larger in the post-TBS1 recording. (3) " $\triangle$ MRBD onset latency" calculated as the MRBD onset latency in the post-TBS1 recording minus the MRBD onset latency in the pre-TBS recording; positive values indicated that the MRBD onset latency was longer in the post-TBS1 recording.

\section{Statistics}

The effect of cTBS on corticospinal excitability was analyzed for statistical significance using a $2 \times 3$ repeated-measures $(\mathrm{RM})$ ANOVA with group (responder, non-responder) as the between-subjects factor and block (pre-TBS, post-TBS1, post-TBS2) as the within-subjects factor. Reaction times were analyzed using a three-way RM ANOVA with group (responders, nonresponders) as the between-subject factors and finger (left, right) and block (pre-TBS, post-TBS1, post-TBS2) as within-subject factors.

The effects of cTBS on spontaneous bilateral M1 oscillatory activity was analyzed using a two-way RM ANOVA with group (responder, non- 

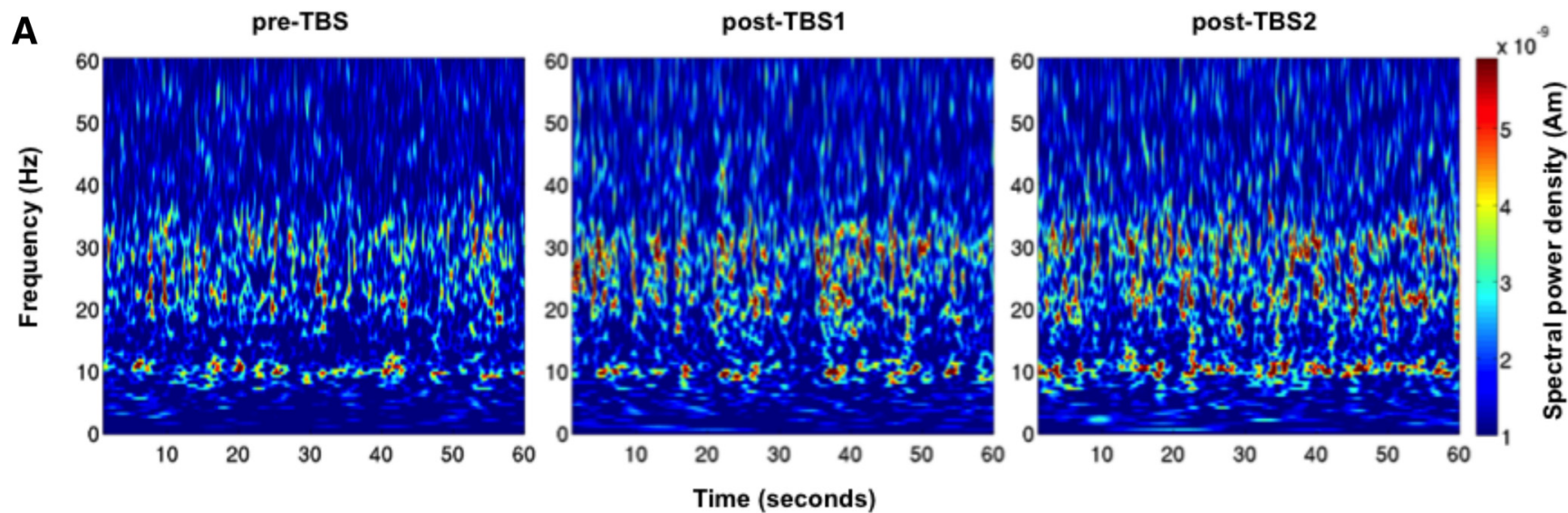

B

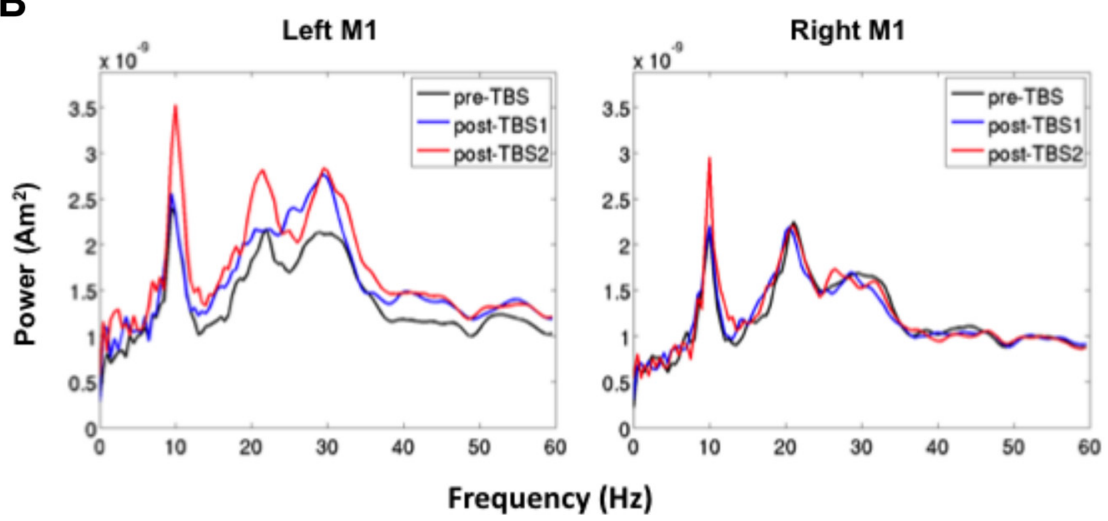

C

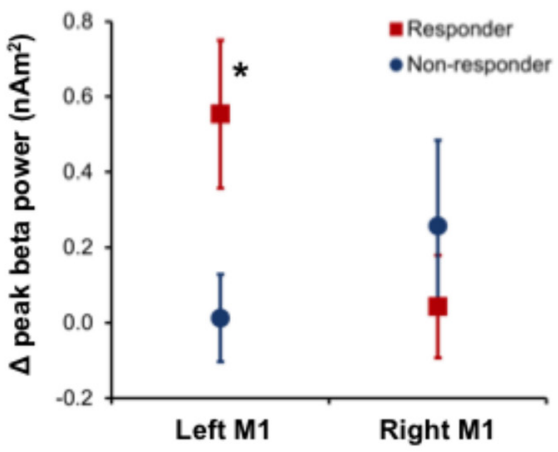

Figure 2. Effect of cTBS on spontaneous oscillatory beta activity. A, Time-frequency representations of spontaneous oscillatory activity recorded from left M1 in a representative responder participant. Each plot represents $60 \mathrm{~s}$ of spontaneous oscillatory activity recorded during each of the three MEG recording blocks. $\boldsymbol{B}$, Power spectral density profiles of both left M1 and right M1 oscillatory activity were then created for each $60 \mathrm{~s}$ period. The peak amplitude of the spontaneous oscillatory power within the beta-band frequency $(15-35 \mathrm{~Hz})$ was obtained from each $60 \mathrm{~s}$ period and averaged across pre-TBS and post-TBS recording blocks. C, Change in oscillatory beta power was calculated as the mean power in the post-TBS minus the mean power in the pre-TBS recording. Data points represent the mean \pm SEM in responder (red squares) and nonresponder (blue circles) participants. ${ }^{*}$ indicates that the peak beta power recorded from left M1 of responder participants was significantly higher after cTBS ( $p=0.012)$.

responder) as the between-subjects factor and hemisphere (left M1, right M1) the within-subject factor. The effects of cTBS on the magnitude and onset latency of the MRBD in the responder participants were analyzed using a two-way RM ANOVA with block (pre-TBS, post-TBS1) and hemisphere (left M1, right M1) as within-subject factors.

The strength of the relationships between $\triangle \mathrm{RT}$ and the $\triangle \mathrm{MRBD}$ magnitude and the $\triangle \mathrm{MRBD}$ onset latency was determined using correlation analyses with Pearson's $r$.

Data points that did not follow a normal distribution were natural log transformed before statistical analysis. When appropriate, main effects and interaction effects resulting from the ANOVAs were further investigated using post hoc $t$ tests. $p$ values $<0.05$ were considered statistically significant. All statistical analysis procedures were performed using SPSS version 20

\section{Results}

Effect of cTBS on corticospinal excitability

Eight of the 16 participants displayed a significant decrease in corticospinal excitability in the 5 to $45 \mathrm{~min}$ following cTBS. The mean reduction of MEP amplitude within each of these individual responder participants (average across all post-cTBS blocks) ranged from 53 to $89 \%$ of the pre-cTBS recording. The remaining eight participants, in whom corticospinal excitability was either unaffected or increased in the 5 to 45 min following cTBS, were categorized as nonresponders (range from 3 to $110 \%$ increase from pre-cTBS). No significant $(p=0.37)$ difference was observed in the mean level of corticospinal excitability recorded in the responder and nonresponder participant groups in pre-TBS block (Fig. 4B). RM ANOVA was used to determine the difference in MEP amplitude between pre-TBS, post-TBS1 (10-25 $\mathrm{min})$, and post-TBS2 (30-45 $\mathrm{min})$ between responder and nonresponder groups. This revealed a significant interaction of group $\times$ block on MEP amplitude $F_{(2,28)}=16.8, p<0.001$. Compared with baseline, MEP amplitudes of the responder participants reduced by $243 \mu \mathrm{V}$ at post-TBS1 $(p=0.03)$ and by 263 $\mu \mathrm{V}$ at post-TBS2 $(p=0.01)$. In contrast, MEP amplitudes of the nonresponder participants had a nonsignificant increase of 179 $\mu \mathrm{V}$ at post-TBS1 $(p=0.13)$ and a significant increase of $308 \mu \mathrm{V}$ at post-TBS2 $(p=0.003)$.

\section{Effect of cTBS on reaction time}

The effect of cTBS, applied over left M1, on mean reaction time was first analyzed with responder and nonresponder participants as separate groups (Fig. $4 C$ ). RM ANOVA revealed a significant interaction of hand $\times$ block $\times$ group $F_{(2,28)}=4.97, p=0.014$. In responder participants, there was a significant increase in mean reaction-time of right index finger abductions during the postTBS1 recording $(22.0 \pm 18.4 \mathrm{~ms}, p=0.045)$, although consistent with previous observations (Huang et al., 2005) this effect was not sustained during the post-TBS2 recording $(1.5 \pm 11.0 \mathrm{~ms} ; p=$ $0.80)$. In contrast, nonresponder participants showed a significant reduction in RT during both post-TBS1 $(11.8 \pm 4.4 \mathrm{~ms}$; 
A

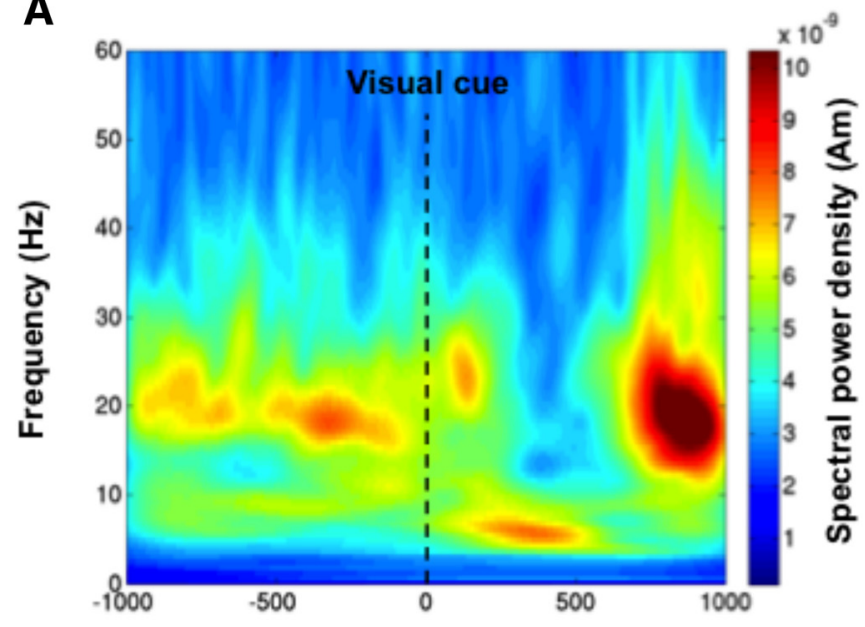

C

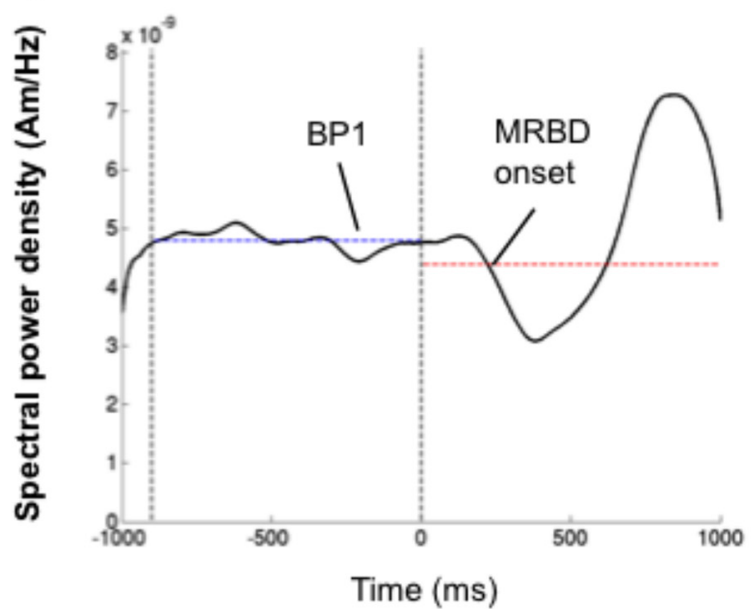

B

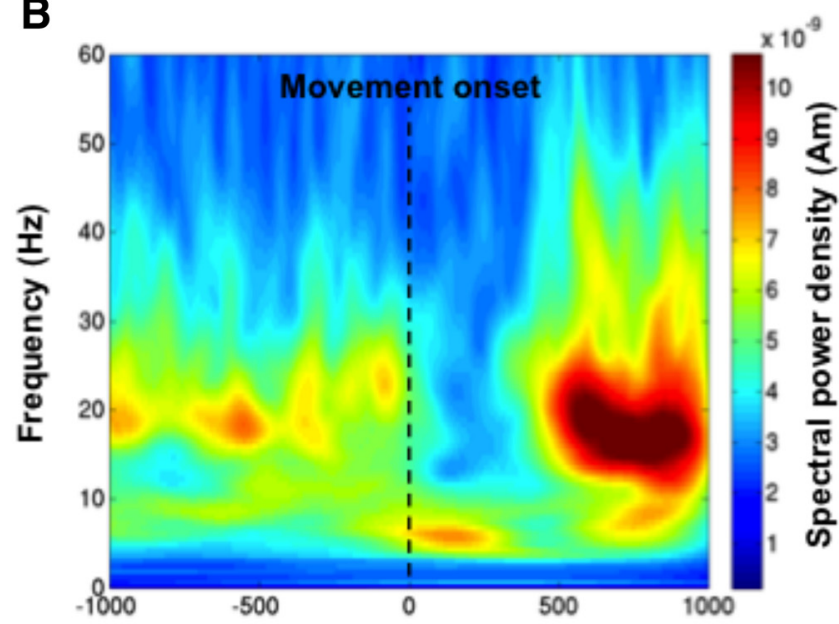

D

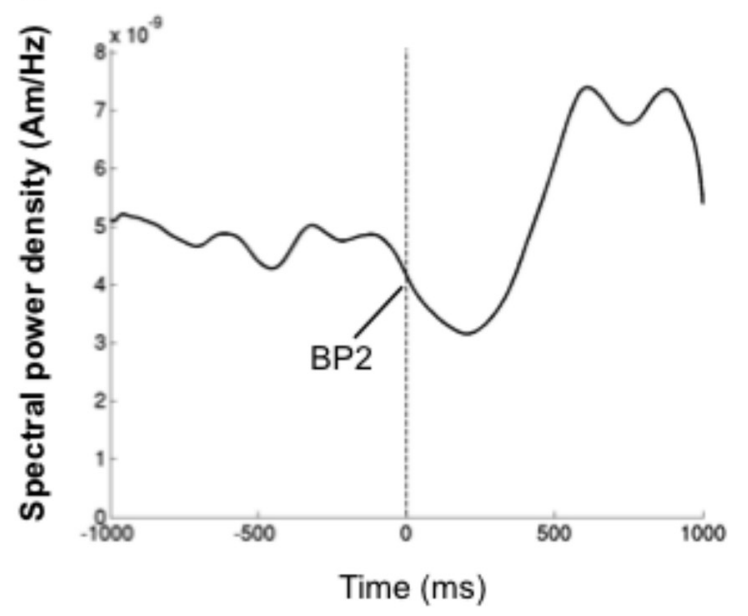

Figure 3. Measurement of movement-related beta activity. $\boldsymbol{A}$, Time-frequency representation of the virtual sensor output from the left M1 of a responder participant as they performed abductions of the right index finger. The movement trials from the pre-TBS recording were aligned from -1.0 to +1.0 s around the onset of the visual go-cue. $\boldsymbol{B}$, Left M1 virtual sensor output as presented in $A$, but realigned from -1.0 to +1.0 s around the movement onset of the right index finger. $C$, Time course of the averaged spectral power in the beta frequency ( $15-35 \mathrm{~Hz}$ ) band aligned to the visual cue. The baseline beta power (BP1), which is represented by the blue horizontal dashed line, was calculated as the mean amplitude during the $900 \mathrm{~ms}$ before the visual cue onset, as represented by the black vertical dashed lines. The onset of the MRBD was also determined as the first point following the visual cue in which the beta-band power fell $<2.5 \mathrm{SD}$ s of BP1. This threshold is represented by the red horizontal dashed line. $\boldsymbol{D}$, Time course of the averaged spectral power in the beta frequency band aligned to the movement onset. The magnitude of the MRBD was calculated by first measuring the power of the beta activity at the movement onset (BP2). To obtain a relative change from baseline this value was then subtracted from the power measured during the baseline period (BP1) in C.

$p=0.001)$ and post-TBS2 $(23.1 \pm 14.1 \mathrm{~ms} ; p=0.015)$ recordings. There were no significant effects of cTBS on mean reactiontimes recorded during left index finger abductions in either responder or nonresponder participants.

\section{Effect of cTBS on resting cortical oscillations}

SAM analysis of the PMBR provided robust localization of bilateral M1 hand area, associated with finger abductions (Fig. 1B). Virtual electrodes, constructed at these sources during the rest periods, revealed the presence of bilateral spontaneous beta activity in all 16 participants. An increase in beta oscillatory power was observed following cTBS in the stimulated hemisphere of responder participants (Fig. $2 A$ ). As demonstrated by the power spectral density profiles (Fig. $2 B$ ) of an individual responder participant, cTBS increased the power of the spontaneous oscillatory beta activity during both post-TBS1 and post-TBS2 MEG recordings. This effect was not detected in the nonstimulated hemisphere. To maximize the power of the statistical analysis, data from both post-TBS recordings were then averaged (Fig. 2C). RM
ANOVA confirmed a significant interaction of hemisphere $\times$ group $F_{(1,14)}=6.32, p=0.025$. The interaction effect was due to the responder participants having a significant increase in left M1 beta-band power from pre-TBS to post-TBS recordings $(p=$ $0.012)$. No such effect was detected in the right M1 of responder participants $(p=0.85)$ or either left M1 $(p=0.88)$ or right M1 $(p=0.12)$ of nonresponder participants.

In contrast to the beta activity, virtual electrodes revealed the presence of clear bilateral $\mathrm{mu}(8-12 \mathrm{~Hz})$ activity peaks in only six of the eight responder participants and five of the eight nonresponder participants. Due to the unequal number of participants in each group, we compared the magnitude of the cTBSmediated change in mu power in left M1 versus right M1 using paired $t$ tests. Results revealed no significant hemispheric difference in mu power change in left $\mathrm{M} 1$ versus right $\mathrm{M} 1$ in either the responder $\left(0.63 \pm 0.83\right.$ vs $\left.0.09 \pm 0.70 \mathrm{nAm}^{2} ; t_{(5)}=1.2, p=0.28\right)$ or nonresponder groups $\left(0.14 \pm 0.19\right.$ vs $0.57 \pm 0.66 \mathrm{nAm}^{2} ; t_{(4)}=$ -2.0, $p=0.11)$. Furthermore, a correlation analysis of the change in power in the beta and mu frequencies over the post- 
cTBS time periods confirmed the independence of these signals in both the responder $\left(r^{2}=0.13, p=0.43\right)$ and nonresponder $\left(r^{2}=0.08, p=0.53\right)$ participants.

\section{Effect of cTBS on MRBD}

Our results indicated that cTBS delayed the reaction time of the right index finger abductions in the post-TBS1 recordings of responder participants (Fig. $4 C$ ). We therefore examined the effects of cTBS on movement-related beta oscillatory activity by focusing on a comparison between the pre-TBS and post-TBS1 recordings of responder participants. We did not examine the effects of cTBS on movementrelated gamma $(60-90 \mathrm{~Hz})$ oscillatory activity as we did not consistently detect the presence of this activity in the pre-TBS recordings. The MRBD was measured as the reduction in the power of the beta activity from the prestimulus period to the onset of movement. During the pre-TBS recordings, the MRBD was $16.4 \pm 7.7 \%$ in left $\mathrm{M} 1$ and $13.1 \pm 9.3 \%$ in right M1 (Fig. $5 A$ ). During the post-TBS1 recordings, the MRBD reduced to $10.7 \pm 3.2 \%$ in left $\mathrm{M} 1$ and $11.5 \pm 6.4 \%$ in right M1; however, RM ANOVA indicated that there were no significant main effects of cTBS,

$F_{(1,7)}=0.42, p=0.54$, or hemisphere, $F_{(1,7)}=2.0, p=0.20$, on the magnitude of the MRBD, nor was there an interaction effect, $F_{(1,7)}=2.2, p=0.18$.

In addition to measuring the percentage magnitude of the MRBD, a threshold crossing procedure was also used to measure the MRBD onset latency (Fig. $3 C$ ). During the pre-TBS recordings, the MRBD onset was $208.9 \pm 53.9 \mathrm{~ms}$ in left M1 and $252.6 \pm$ $39.3 \mathrm{~ms}$ in right M1 (Fig. 5B). During the post-TBS1 recordings, the MRBD onset latency increased to $247.2 \pm 79.3 \mathrm{~ms}$ in left M1 and $263.6 \pm 74.3 \mathrm{~ms}$ in right $\mathrm{M} 1$; RM ANOVA indicated that there were no significant main effects of cTBS, $F_{(1,7)}=1.8, p=$ 0.23 , or hemisphere, $F_{(1,7)}=1.2, p=0.32$, on the MRBD onset latency, nor was there an interaction effect, $F_{(1,7)}=0.5, p=0.52$.

Correlation analyses were then performed to determine whether the cTBS-induced changes in reaction time from the right index finger were associated with changes in oscillatory beta activity recorded from left M1 (Fig. $5 C, D$ ). The $\Delta$ RT following cTBS did not show a significant correlation with either the $\Delta$ MRBD magnitude $\left(r^{2}=0.17, p=0.30\right)$ or the $\Delta$ MRBD onset latency $\left(r^{2}=0.12, p=0.78\right)$.

\section{Discussion}

Following the protocol of Huang et al. (2005), cTBS applied over left M1 reduced corticospinal excitability in 50\% of participants. Consistent with recent findings (McAllister et al., 2011; Goldsworthy et al., 2012; Hamada et al., 2012), the inhibitory after-effects of cTBS show high interparticipant variability; similar to that found with $1 \mathrm{~Hz}$ rTMS protocols (Fitzgerald et al., 2006). Importantly, our MEG source-level recordings demonstrate that this variability is associated with changes in M1 neuronal network synchrony. Specifically, we observed an increase in spontaneous beta power in stimulated M1, in a group of partici-
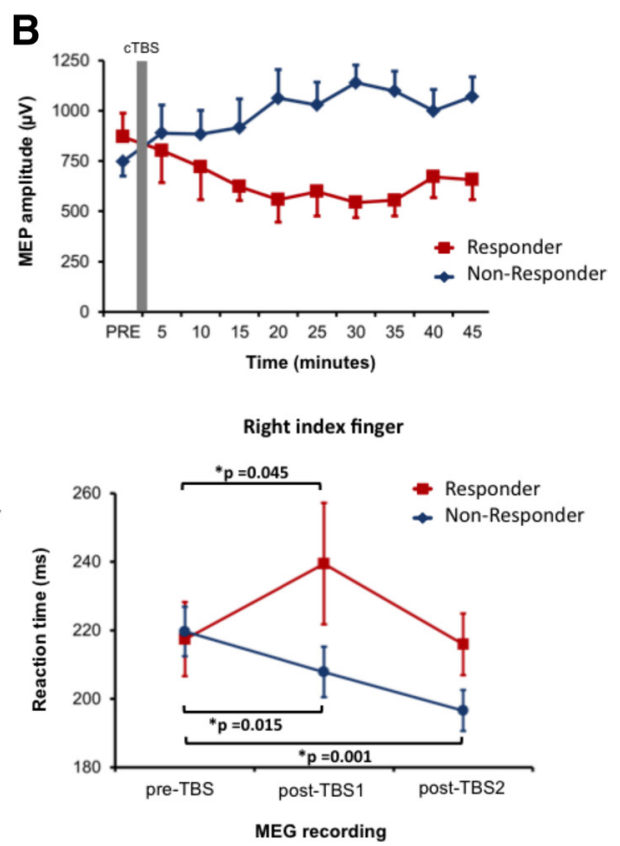

EG recording

TMS MEP

1 M
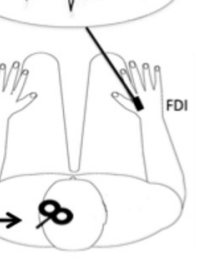

eft index finger

- Responder

- Non-Responder

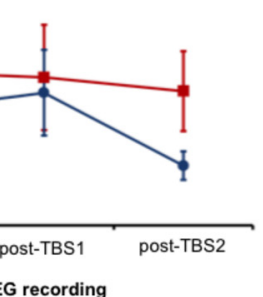

B

Figure 4. Effect of CTBS on corticospinal excitability and reaction times. $\boldsymbol{A}, \mathrm{cTBS}$ was applied over left M1 at an intensity of $80 \%$ 列

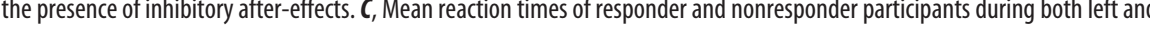
index finger abductions in each MEG recording. Data points represent the mean $\pm S E M .{ }^{*}$ indicates significant difference $(p<0.05)$ in reaction time between the two highlighted conditions.

pants that display reduced corticospinal excitability and increased reaction time following cTBS. To our knowledge, this is the first study to identify and characterize individual variability of oscillatory activity in response to cTBS.

In the context of genetic evidence underlying cTBS efficacy, an attractive proposition is that brain-derived neurotrophic factor (BDNF) underlies the differential response between responders and nonresponders. Cheeran et al. (2008) showed that cTBSreduction in corticospinal excitability is associated with the Val66Val polymorphism. Importantly, BDNF modulation of postsynaptic GABA-A responses in the cortex (Tanaka et al., 1997) is likely to directly shape the GABAergic interneurondriven beta oscillations in M1 (Yamawaki et al., 2008). Given that this phasic inhibitory signature underlies the spontaneous beta signal observed with MEG (Jensen et al., 2005), which is elevated in power following GABA-A modulation (Hall et al., 2010), it is unsurprising that spontaneous beta power underlies the distinction between responder and nonresponder participants. A metaanalysis conducted by (Gratacòs et al., 2007) estimated the frequency of the Val-allele to be $\sim 81 \%$ in Caucasian and $56 \%$ in Asian ethnic groups, respectively. However, with six Caucasian and two Asian participants in both our responder and nonresponder groups, and without genetic information, we can only speculate on the prevalence of the Val-allele polymorphism and its association with inhibitory after-effects and increased beta power following cTBS.

Our observation of increased spontaneous M1 beta power is consistent with the results of Stagg et al. (2009) who found that MRS-measured GABA concentration within M1 increased following cTBS over this region, and such M1 GABA concentrations have been shown previously to correlate with PMBR power (Gaetz et al., 2011). These findings indicate that inhibitory after- 
A
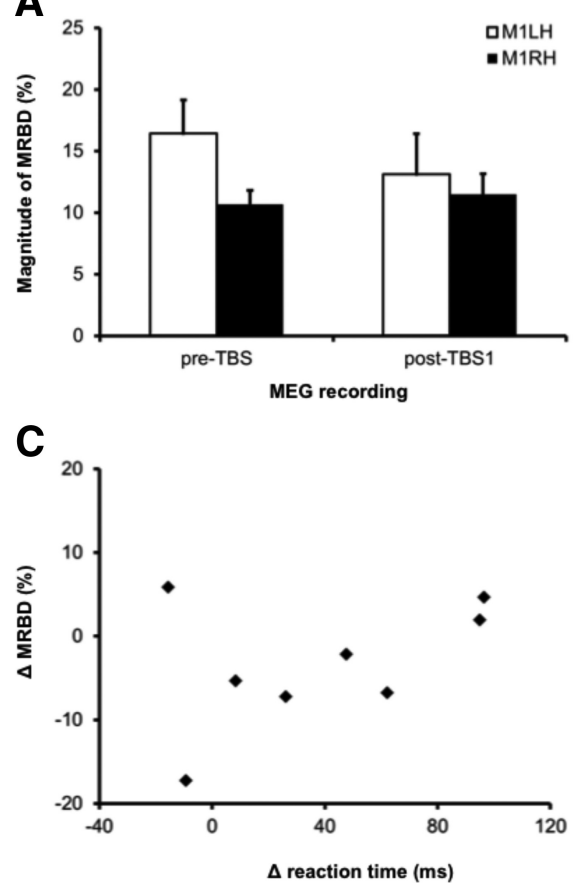

B

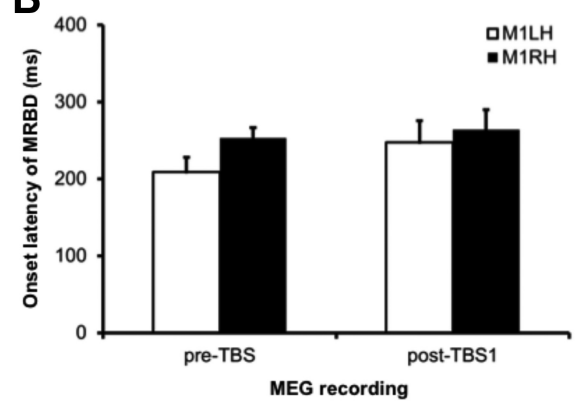

D

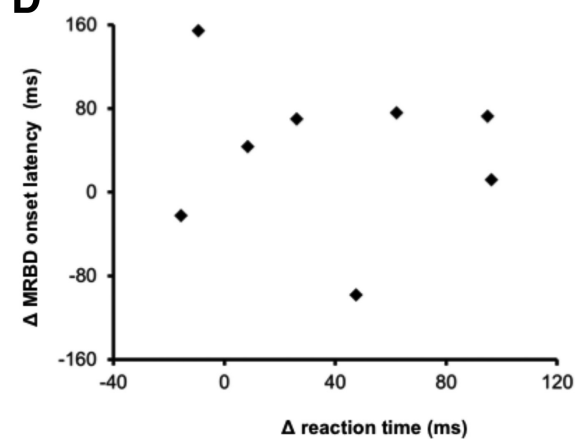

Figure 5. Effect of cTBS on movement-related oscillatory beta activity. $\boldsymbol{A}$, Percentage magnitude of the MRBD measured from both left and right M1 during abduction of the right index finger in the pre-TBS and post-TBS1 recordings. $\boldsymbol{B}$, Onset latency of the MRBD measured from both left and right M1 during abduction of the right index finger in the pre-TBS and post-TBS1 recordings. $C$, The relationship between the change in the power of MRBD from left M1 following cTBS plotted against the change in reaction time in the right index finger. Positive values indicate that reaction times ( $y$-axis) or percentage MRBD magnitude ( $x$-axis) were longer in the post-TBS1 recording as compared with the pre-TBS recording. $D$, The change in the onset latency of the MRBD from left M1 following CTBS plotted against the change in reaction time of the right index finger. Positive values indicate that reaction times ( $y$-axis) or MRBD onset latency ( $x$-axis) were longer in the post-TBS1 recording as compared with the pre-TBS recording.

effects of cTBS on M1 activity may be GABAergically mediated. Evidence from rat models indicates that TBS affects protein expression related to GABA synthesis (Trippe et al., 2009; Benali et al., 2011; Funke and Benali, 2011), consistent with the sustained neuroplastic effects. However, given that GABA-mediated phasic inhibition exerts its effects through entrainment of glutamatergic pyramidal cells, these elements of the circuit should not be overlooked. Indeed, cTBS effects are blocked in the presence of the $\mathrm{N}$-methyl-D-aspartate antagonist memantine (Huang et al., 2007).

EEG recordings following single-pulse TMS demonstrate a negative association between the spontaneous beta power and contralateral MEP amplitude (Zarkowski et al., 2006; Mäki and Ilmoniemi, 2010). This is consistent with the increase in spontaneous beta power in stimulated M1 following cTBS. Corticospinal excitability is reduced in the 200 to $1000 \mathrm{~ms}$ following stimulation of the median nerve (Chen et al., 1999) and from 500 to $1000 \mathrm{~ms}$ after a simple finger movement (Chen et al., 1998), corresponding with increases in beta power found during these tasks (Salenius et al., 1997; Jurkiewicz et al., 2006). These data support the view that elevated M1 beta power is related to cortical inhibition, consistent with the cortical-idling hypothesis (Pfurtscheller, 1992), but slightly less congruous with the hypothesis of beta as a promoter of tonic activity (Brown, 2007).

Our results demonstrated the presence of a clear peak within the $\mathrm{mu}(8-12 \mathrm{~Hz})$ frequency band in 11 of the 16 participants. The changes in mu power following cTBS in both responders and nonresponders proved nonsignificant and we have therefore focused our discussion on beta power changes. However, the low

number of participants exhibiting $\mathrm{mu}$ in the present cohort suggest that this feature would be worthy of further investigation in future studies.

McAllister et al. (2011), using EEG sensor analysis over electrode site C3, found no association between spontaneous beta power $(14-30 \mathrm{~Hz})$ and the magnitude of corticospinal inhibition following $40 \mathrm{~s}$ of cTBS over left M1. Furthermore, no change in spontaneous beta power, as measured during the $8 \mathrm{~min}$ post-stimulation, was detected. In contrast, Noh et al. (2012) reported that $20 \mathrm{~s}$ of cTBS applied over left M1, produced widespread increases in low beta (13-20 $\mathrm{Hz}$ ) power in both stimulated and nonstimulated hemispheres. Our current results are in partial agreement with Noh et al. (2012), as we only observed increased beta activity in the stimulated M1 region, possibly a consequence of different recording techniques. Our source-level MEG approach provides a more sensitive reconstruction of M1 activity, as it accounts for interparticipant variations in recording location. The use of MEG also avoids the problem of signal contamination from a cortical reference site, often seen with EEG.

Huang et al. (2005) also reported that cTBS delayed simple reaction time in the contralateral but not ipsilateral hand. This effect was present at $10 \mathrm{~min}$ but not 30 min poststimulation. Some subsequent studies have failed to detect an effect of M1 cTBS on simple or choice reaction time (Schabrun et al., 2008; Noh et al., 2012); however, others have reported that cTBS can disrupt grip force (Nowak et al., 2005; Schabrun et al., 2008; Stefan et al., 2008). Here, we expand on previous findings by distinguishing between responder and nonresponder participants. Reaction times of the responders were significantly slower in the contralateral index finger at $10 \mathrm{~min}$ but not $30 \mathrm{~min}$, following the application of cTBS. Nonresponders showed a reduction in reaction time following TBS, which may reflect a facilitatory effect of the stimulation. However, it is also possible that this reduction in reaction time occurs across the experiment due to practice effects as described previously (Noh et al., 2012). Importantly, when considered in the context of nonresponder results, reaction-time of the responder group remains comparatively elevated.

In the context of pathology, PD is typically associated with an emergence of elevated "pathological" beta oscillations in cortical areas (Sharott et al., 2005; Mallet et al., 2008; Pollok et al., 2012) and subcortical areas (Brown et al., 2001; Cassidy et al., 2002; Sharott et al., 2005; Mallet et al., 2008). The importance of beta activity in PD is endorsed by the observation of attenuated beta power and coherence accompanying symptomatic relief following treatment with Levodopa or deep brain stimulation (Silberstein et al., 2005; Kühn et al., 2009). In PD patients and healthy controls, elevated beta power in the human motor system is considered antikinetic (Jenkinson and Brown, 2011). The application of transcranial alternating current stimulation at beta frequencies over human M1 has been reported to slow voluntary 
movement and modify force production during visuomotor tasks (Pogosyan et al., 2009; Joundi et al., 2012).

Recordings of local field-potentials from the subthalamic nucleus of PD patients show a positive correlation between the latency of MRBD and reaction time (Kühn et al., 2004; Ray et al., 2012). These results led to our hypothesis that cTBS-mediated increases in reaction time would be accompanied by a concomitant increases in the onset latency of the MRBD. We observed that cTBS increased reaction times in the contralateral hand. However, although the onset latency of MRBD increased by $\sim 40 \mathrm{~ms}$, the variance in the sample rendered this result nonsignificant. Similarly, despite an increase in spontaneous beta power, no significant difference in MRBD amplitude was seen at EMG onset. This finding was unexpected. However, previous studies have also demonstrated that manipulations that increase the amplitude of MRBD are not necessarily accompanied by a change in function measureable with reaction time (Hall et al., 2011; Muthukumaraswamy et al., 2012). In contrast, Noh et al. (2012) reported that cTBS increased the power of the $\alpha$ and low beta power recorded during finger movements. These effects are rather difficult to interpret, as all phases of the movement period, including the MRBD and PMBR, from both ipsilateral and contralateral movements were averaged together. In a related MEG study, Hsu et al. (2011) reported that the excitatory after-effects of intermittent TBS were associated with increased PMBR amplitudes in the stimulated M1 and decreased PMBR amplitudes in the nonstimulated M1 hemisphere. However, this finding is difficult to interpret because TBS-mediated changes in spontaneous M1 beta activity were not examined and the PMBR changes were calculated relative to this baseline. Gamma synchronization, which occurs at movement onset, has been reported previously (Muthukumaraswamy, 2010). This short-lived oscillatory increase was not consistently observed in our participants, perhaps due to the low trial number. We were, therefore, unable to determine whether movement-related gamma activity was modified by cTBS, but suggest that this would be worthy of further exploration.

While noninvasive brain-stimulation techniques can produce sustained neuroplastic effects and modify motor behavior, high levels of interindividual variability limit their potential therapeutic value (Ridding and Rothwell, 2007). We demonstrate that the combined use of TMS and source-level MEG recordings provides a discriminatory method for examining this phenomenon. Specifically, with $50 \%$ of participants in this study exhibiting changes in corticospinal excitability and reaction time following cTBS, we were able to characterize these individual differences. Our results demonstrate that the observed variability may relate to GABAergic mechanisms governing the presence of oscillatory beta activity within M1. Further research on this topic is therefore likely to provide important new data to improve future interventions and treatment.

\section{References}

Adjamian P, Barnes GR, Hillebrand A, Holliday IE, Singh KD, Furlong PL, Harrington E, Barclay CW, Route PJ (2004) Co-registration of magnetoencephalography with magnetic resonance imaging using bite-barbased fiducials and surface-matching. Clin Neurophysiol 115:691-698. CrossRef Medline

Benali A, Trippe J, Weiler E, Mix A, Petrasch-Parwez E, Girzalsky W, Eysel UT, Erdmann R, Funke K (2011) Theta-burst transcranial magnetic stimulation alters cortical inhibition. J Neurosci 31:1193-1203. CrossRef Medline

Brown P (2007) Abnormal oscillatory synchronisation in the motor system leads to impaired movement. Curr Opin Neurobiol 17:656-664. CrossRef Medline
Brown P, Oliviero A, Mazzone P, Insola A, Tonali P, Di Lazzaro V (2001) Dopamine dependency of oscillations between subthalamic nucleus and pallidum in Parkinson's disease. J Neurosci 21:1033-1038. Medline

Cassidy M, Mazzone P, Oliviero A, Insola A, Tonali P, Di Lazzaro V, Brown P (2002) Movement-related changes in synchronization in the human basal ganglia. Brain 125:1235-1246. CrossRef Medline

Cheeran B, Talelli P, Mori F, Koch G, Suppa A, Edwards M, Houlden H, Bhatia K, Greenwood R, Rothwell JC (2008) A common polymorphism in the brain-derived neurotrophic factor gene (BDNF) modulates human cortical plasticity and the response to rTMS. J Physiol 586:5717-5725. CrossRef Medline

Chen R, Yaseen Z, Cohen LG, Hallett M (1998) Time course of corticospinal excitability in reaction time and self-paced movements. Ann Neurol 44: 317-325. CrossRef Medline

Chen R, Corwell B, Hallett M (1999) Modulation of motor cortex excitability by median nerve and digit stimulation. Exp Brain Res 129:77-86. CrossRef Medline

Di Lazzaro V, Pilato F, Saturno E, Oliviero A, Dileone M, Mazzone P, Insola A, Tonali PA, Ranieri F, Huang YZ, Rothwell JC (2005) Theta-burst repetitive transcranial magnetic stimulation suppresses specific excitatory circuits in the human motor cortex. J Physiol 565:945-950. CrossRef Medline

Eggers C, Fink GR, Nowak DA (2010) Theta burst stimulation over the primary motor cortex does not induce cortical plasticity in Parkinson's disease. J Neurol 257:1669-1674. CrossRef Medline

Fitzgerald PB, Fountain S, Daskalakis ZJ (2006) A comprehensive review of the effects of rTMS on motor cortical excitability and inhibition. Clin Neurophysiol 117:2584-2596. CrossRef Medline

Funke K, Benali A (2011) Modulation of cortical inhibition by rTMSfindings obtained from animal models. J Physiol 589:4423-4435. Medline

Gaetz W, Edgar JC, Wang DJ, Roberts TP (2011) Relating MEG measured motor cortical oscillations to resting $\gamma$-aminobutyric acid (GABA) concentration. Neuroimage 55:616-621. CrossRef Medline

Gentner R, Wankerl K, Reinsberger C, Zeller D, Classen J (2008) Depression of human corticospinal excitability induced by magnetic theta-burst stimulation: evidence of rapid polarity-reversing metaplasticity. Cereb Cortex 18:2046-2053. Medline

Goldsworthy MR, Pitcher JB, Ridding MC (2012) The application of spaced theta burst protocols induces long-lasting neuroplastic changes in the human motor cortex. Eur J Neurosci 35:125-134. CrossRef Medline

Gratacòs M, González JR, Mercader JM, de Cid R, Urretavizcaya M, Estivill X (2007) Brain-derived neurotrophic factor Val66Met and psychiatric disorders: meta-analysis of case-control studies confirm association to substance-related disorders, eating disorders, and schizophrenia. Biol Psychiatry 61:911-922. CrossRef Medline

Hall SD, Barnes GR, Furlong PL, Seri S, Hillebrand A (2010) Neuronal network pharmacodynamics of GABAergic modulation in the human cortex determined using pharmaco-magnetoencephalography. Hum Brain Mapp 31:581-594. Medline

Hall SD, Stanford IM, Yamawaki N, McAllister CJ, Rönnqvist KC, Woodhall GL, Furlong PL (2011) The role of GABAergic modulation in motor function related neuronal network activity. Neuroimage 56:1506-1510. CrossRef Medline

Hamada M, Murase N, Hasan A, Balaratnam M, Rothwell JC (2012) The Role of Interneuron Networks in Driving Human Motor Cortical Plasticity. Cereb Cortex. Advance online publication. Retrieved June 1, 2012. doi:10.1093/cercor/bhs147. CrossRef

Hess G, Donoghue JP (1996) Long-term depression of horizontal connections in rat motor cortex. Eur J Neurosci 8:658-665. CrossRef Medline

Hillebrand A, Singh KD, Holliday IE, Furlong PL, Barnes GR (2005) A new approach to neuroimaging with magnetoencephalography. Hum Brain Mapp 25:199-211. CrossRef Medline

Hoogendam JM, Ramakers GM, Di Lazzaro V (2010) Physiology of repetitive transcranial magnetic stimulation of the human brain. Brain Stimul 3:95-118. CrossRef Medline

Hsu YF, Liao KK, Lee PL, Tsai YA, Yeh CL, Lai KL, Huang YZ, Lin YY, Lee IH (2011) Intermittent theta burst stimulation over primary motor cortex enhances movement-related beta synchronisation. Clin Neurophysiol 122:2260-2267. CrossRef Medline

Huang YZ, Edwards MJ, Rounis E, Bhatia KP, Rothwell JC (2005) Theta burst stimulation of the human motor cortex. Neuron 45:201-206. CrossRef Medline 
Huang YZ, Chen RS, Rothwell JC, Wen HY (2007) The after-effect of human theta burst stimulation is NMDA receptor dependent. Clin Neurophysiol 118:1028-1032. CrossRef Medline

Huang YZ, Rothwell JC, Edwards MJ, Chen RS (2008) Effect of physiological activity on an NMDA-dependent form of cortical plasticity in human. Cereb Cortex 18:563-570. Medline

Jenkinson N, Brown P (2011) New insights into the relationship between dopamine, beta oscillations and motor function. Trends Neurosci 34: 611-618. CrossRef Medline

Jensen O, Goel P, Kopell N, Pohja M, Hari R, Ermentrout B (2005) On the human sensorimotor-cortex beta rhythm: sources and modeling. Neuroimage 26:347-355. CrossRef Medline

Joundi RA, Jenkinson N, Brittain JS, Aziz TZ, Brown P (2012) Driving oscillatory activity in the human cortex enhances motor performance. Curr Biol 22:403-407. CrossRef Medline

Jurkiewicz MT, Gaetz WC, Bostan AC, Cheyne D (2006) Post-movement beta rebound is generated in motor cortex: evidence from neuromagnetic recordings. Neuroimage 32:1281-1289. CrossRef Medline

Keel JC, Smith MJ, Wassermann EM (2001) A safety screening questionnaire for transcranial magnetic stimulation. Clin Neurophysiol 112:720. Medline

Kühn AA, Tsui A, Aziz T, Ray N, Brücke C, Kupsch A, Schneider GH, Brown P (2009) Pathological synchronisation in the subthalamic nucleus of patients with Parkinson's disease relates to both bradykinesia and rigidity. Exp Neurol 215:380-387. CrossRef Medline

Kühn A, Williams D, Kupsch A, Limousin P, Hariz M, Schneider GH, Yarrow K, Brown P (2004) Event-related beta desynchronization in human subthalamic nucleus correlates with motor performance. Brain 127:735-746. CrossRef Medline

Mallet N, Pogosyan A, Sharott A, Csicsvari J, Bolam JP, Brown P, Magill PJ (2008) Disrupted dopamine transmission and the emergence of exaggerated beta oscillations in subthalamic nucleus and cerebral cortex. J Neurosci 28:4795-4806. CrossRef Medline

Mäki H, Ilmoniemi RJ (2010) EEG oscillations and magnetically evoked motor potentials reflect motor system excitability in overlapping neuronal populations. Clin Neurophysiol 121:492-501. CrossRef Medline

McAllister SM, Rothwell JC, Ridding MC (2011) Cortical oscillatory activity and the induction of plasticity in the human motor cortex. Eur J Neurosci 33:1916-1924. CrossRef Medline

Muthukumaraswamy SD (2010) Functional Properties of Human Primary Motor Cortex Gamma Oscillations. J Neurophysiol 104:2873-2885. CrossRef Medline

Muthukumaraswamy SD, Myers JF, Wilson SJ, Nutt DJ, Lingford-Hughes A, Singh KD, Hamandi K (2012) The effects of elevated endogenous GABA levels on movement-related network oscillations. Neuroimage 66C:36-41. Medline

Noh NA, Fuggetta G, Manganotti P, Fiaschi A (2012) Long lasting modulation of cortical oscillations after continuous theta burst transcranial magnetic stimulation. PloS One 7:e35080. CrossRef Medline

Nowak DA, Voss M, Huang YZ, Wolpert DM, Rothwell JC (2005) Highfrequency repetitive transcranial magnetic stimulation over the hand area of the primary motor cortex disturbs predictive grip force scaling. Eur J Neurosci 22:2392-2396. CrossRef Medline

Pfurtscheller G (1992) Event-related synchronisation (ERS): an electrophysiological correlate of cortical areas at rest. Electroencephalogr Clin Neurophysiol 83:62-69. CrossRef Medline

Pogosyan A, Gaynor LD, Eusebio A, Brown P (2009) Boosting cortical activity at Beta-band frequencies slows movement in humans. Curr Biol 19:1637-1641. CrossRef Medline
Pollok B, Krause V, Martsch W, Wach C, Schnitzler A, Südmeyer M (2012) Motor-cortical oscillations in early stages of Parkinson's disease. J Physiol 590:3203-3212. CrossRef Medline

Ray NJ, Brittain JS, Holland P, Joundi RA, Stein JF, Aziz TZ, Jenkinson N (2012) The role of the subthalamic nucleus in response inhibition: evidence from local field potential recordings in the human subthalamic nucleus. Neuroimage 60:271-278. Medline

Ridding MC, Rothwell JC (2007) Is there a future for therapeutic use of transcranial magnetic stimulation? Nat Rev Neurosci 8:559-567. CrossRef Medline

Robinson SE, Rose DF (1992) Current source estimation by spatially filtered MEG. In: Biomagnetism: clinical aspects (Romani G, ed), pp 761-765. Amsterdam: Excerpta Medica.

Salenius S, Schnitzler A, Salmelin R, Jousmäki V, Hari R (1997) Modulation of human cortical rolandic rhythms during natural sensorimotor tasks. Neuroimage 5:221-228. CrossRef Medline

Schabrun SM, Ridding MC, Miles TS (2008) Role of the primary motor and sensory cortex in precision grasping: a transcranial magnetic stimulation study. Eur J Neurosci 27:750-756. CrossRef Medline

Sharott A, Magill PJ, Harnack D, Kupsch A, Meissner W, Brown P (2005) Dopamine depletion increases the power and coherence of betaoscillations in the cerebral cortex and subthalamic nucleus of the awake rat. Eur J Neurosci 21:1413-1422. CrossRef Medline

Silberstein P, Pogosyan A, Kühn AA, Hotton G, Tisch S, Kupsch A, DowseyLimousin P, Hariz MI, Brown P (2005) Cortico-cortical coupling in Parkinson's disease and its modulation by therapy. Brain 128:1277-1291. CrossRef Medline

Stagg CJ, Wylezinska M, Matthews PM, Johansen-Berg H, Jezzard P, Rothwell JC, Bestmann S (2009) Neurochemical effects of theta burst stimulation as assessed by magnetic resonance spectroscopy. J Neurophysiol 101: 2872-2877. CrossRef Medline

Stefan K, Gentner R, Zeller D, Dang S, Classen J (2008) Theta-burst stimulation: remote physiological and local behavioral after-effects. Neuroimage 40:265-274. CrossRef Medline

Talelli P, Wallace A, Dileone M, Hoad D, Cheeran B, Oliver R, VandenBos M, Hammerbeck U, Barratt K, Gillini C, Musumeci G, Boudrias MH, Cloud GC, Ball J, Marsden JF, Ward NS, Di Lazzaro V, Greenwood RG, Rothwell JC (2012) Theta burst stimulation in the rehabilitation of the upper limb: a semirandomized, placebo-controlled trial in chronic stroke patients. Neurorehabil Neural Repair 26:976-987. CrossRef Medline

Tanaka T, Saito H, Matsuki N (1997) Inhibition of GABA A synaptic responses by brain-derived neurotrophic factor (BDNF) in rat hippocampus. J Neurosci 17:2959-2966. Medline

Trippe J, Mix A, Aydin-Abidin S, Funke K, Benali A (2009) $\theta$ burst and conventional low-frequency rTMS differentially affect GABAergic neurotransmission in the rat cortex. Exp Brain Res 199:411-421. CrossRef Medline

Van Veen BD, van Drongelen W, Yuchtman M, Suzuki A (1997) Localization of brain electrical activity via linearly constrained minimum variance spatial filtering. IEEE Trans Biomed Eng 44:867-880. CrossRef Medline

Vrba J, Robinson SE (2001) Signal processing in magnetoencephalography. Methods 25:249-271. CrossRef Medline

Yamawaki N, Stanford IM, Hall SD, Woodhall GL (2008) Pharmacologically induced and stimulus evoked rhythmic neuronal oscillatory activity in the primary motor cortex in vitro. Neuroscience 151:386-395. CrossRef Medline

Zarkowski P, Shin CJ, Dang T, Russo J, Avery D (2006) EEG and the variance of motor evoked potential amplitude. Clin EEG Neurosci 37:247251. CrossRef Medline 